\title{
ANÁLISE BAYESIANA DO ÍNDICE PERÍMETRO ESCROTAL/PESO DE ANIMAIS NELORE DO ESTADO DO TOCANTINS, BRASIL
}

\author{
BAYESIAN ANALYSIS FOR THE INDEX SCROTAL CIRCUMFERENCE/WEIGHT ON \\ NELLORE ANIMALS BORN IN THE TOCANTINS STATE, BRAZIL
}

\begin{abstract}
Marcondes, C.R. ${ }^{1 *}$, Araújo, R.O. ${ }^{2}$, Vozzi, P.A. ${ }^{3}$, Gunski, R.J., Garnero, A. del V. ${ }^{4}$ e Lôbo, R.B. ${ }^{5}$
${ }^{1}$ Embrapa Pecuária Sudeste. São Carlos. SP. Brasil. *cintia@cppse.embrapa.br

2UnB. Brasília-DF. Brasil. ronyereo@yahoo.com.br

${ }^{3}$ Instituto Nacional de Tecnología Agropecuaria (INTA). EEA Chubut. Argentina. avozzi@chubut.inta.gov.ar ${ }^{4}$ Ciências Biológicas/UNIPAMPA. Campus de São Gabriel/RS. Brasil. rgunski@yahoo.com.br; analiagarnero@yahoo.com.br

${ }^{5}$ Associação Nacional de Criadores e Pesquisadores. ANCP. Ribeirão Preto-SP.Brasil. raysildo@ancp.org.br
\end{abstract}

\section{PALAVRAS CHAVE ADICIONAIS}

Bovinos de corte. Critério alternativo de seleção. Parâmetros genéticos.

\section{RESUMO}

Objetivou-se com este trabalho estimar os parâmetros genéticos do índice perímetro escrotal/ peso padronizado para 365 dias de idade (IND365), via inferência bayesiana, sob dois modelos de análise distintos, em animais da raça Nelore criados no Estado do Tocantins, Brasil. O arquivo final estava constituído de 693 registros do IND365 distribuídos em 16 grupos de contemporâneos. Os componentes de variância foram analisados via inferência bayesiana, por meio de programas em linguagem Fortran. Amostragem a cada 100 ou 500 rodadas e um burn-in pouco conservador poderão ser implementados. As médias pontuais para os coeficientes de herdabilidade direta e materna para o IND365 foram respectivamente: 0,18 (com ou sem efeito materno) e 0,14 . Estes resultados sugerem que o IND365 possa ser utilizado como critério alternativo nos programas de melhoramento e responder de forma satisfatória ao processo de seleção.

\section{SUMMARY}

The aim of this study was to estimate the genetic parameters of the index scrotal circumference/weight standarized for 365 days of age (IND365), via bayesian inference, in two separate models of analysis on Nellore animals born in the

\section{AdDitional KEYWORDS}

Alternative selection criteria. Beef cattle. Genetic parameters.

Tocantins state, Brazil. The file was composed of 693 records of the IND365 divided into 16 contemporaneous group. Variance components were analyzed via bayesian inference, through the programs in Fortran language. Sampling of every 100 or 500 rounds and a little conservative burn-in can be implemented. Means of direct heritabilities were 0.18 (with and without maternal effect) and 0.14 for maternal heritabilities. These results suggest that the IND365 can be used as alternative criterion in breeding programs to Nellore cattle and respond satisfactorily to the selection process.

\section{INTRODUÇÃO}

Nas duas últimas décadas, vários programas de melhoramento genético animal foram implementados no Brasil voltados para as raças bovinas de corte, em especial para a Nelore. Esses programas, normalmente, utilizam como critérios de seleção, características de crescimento e de fertilidade (Pereira et al., 2000; Cyrillo et al., 2004; Yokoo et al., 2007; Boligon et al., 2008). Dentre as características fenotípicas, a taxa de crescimento corporal dos animais 


\section{MARCONDES, ARAÚJO, VOZZI, GUNSKI, GARNERO E LÔBO}

e sua eficiência reprodutiva são dois fatores determinantes para a lucratividade do sistema (Mercadante et al., 1997; Marcondes, 1999). Neste mesmo foco, o Estado do Tocantins vem se destacando maiormente através da pecuária de corte, propiciando a expansão econômica, principalmente com a introdução de atividades agropecuárias em processo de aumento da tecnificação, após o período de simples ocupação territorial como fronteira agrícola. O rebanho tocantinense tem cerca de oito milhões de animais voltados, sobretudo, para o abate realizado em 15 frigoríficos, que juntos somam capacidade para o abate mensal de mais de 165 mil animais. Destes frigoríficos, dois estão na lista geral de exportações e outros poderão se adaptar às exigências internacionais em breve. Com a sanidade do rebanho bovino reconhecida, a carne tocantinense chega aos mercados mais exigentes do mundo, ampliando as fronteiras comerciais do Tocantins. Em 2007, o Estado comercializava carne para mais de 20 países, com destaque para os continentes asiático, europeu e africano (Seagro, 2007), sendo este o principal produto de exportação (Seagro, 2010).

A pecuária nacional, assim como a tocantinense, cresceu expressivamente em termos de exportações, $22,86 \%$ e $489 \%$, respectivamente. Segundo a Secretaria da Agricultura, Pecuária e Abastecimento do Estado do Tocantins, em 2006, foram exportadas 24 mil toneladas de carne bovina, o que rendeu mais de 53 milhões de dólares e muitos benefícios para o povo tocantinense (Seagro, 2007). E esta realidade direciona os pecuaristas a uma tendência que busca incrementar sua atividade por meio de inovações tecnológicas que permitam a obtenção de melhor renda em menor tempo e com menos gastos, principalmente com respeito à maior taxa de lotação das pastagens, aumentando a eficiência das mesmas. Neste sentido é imprescindível, na elaboração de programas de seleção, o conhecimento dos fatores que influenciam o crescimento dos animais, bem como das correlações genéticas entre eles, pois esses parâmetros genéticos constituem elementos básicos para o estabelecimento de diretrizes que possam orientar o melhoramento genético dos animais (Martins Filho et al., 2005). Estudos feitos por Gunski et al. (2001), mostraram que as estimativas de parâmetros genéticos para características ponderais na raça Nelore têm apresentado grande variabilidade, sendo um bom indicador da resposta à seleção ou melhoramento genético a ser alcançado. Estes parâmetros são necessários para estimar as respostas diretas e correlacionadas à seleção, para elaborar índices de seleção, predizer o valor genético dos animais e estudar os efeitos das interações genótipo-ambiente. Com base na necessidade de trazer conhecimentos específicos sobre os rebanhos da raça Nelore criados no Estado do Tocantins, dois projetos de pesquisa foram financiados pelo Conselho Nacional de Desenvolvimento Científico e Tecnológico (CNPq), nas modalidades Jovem Pesquisador e Desenvolvimento Científico Regional-DCR, com apoio da Secretaria de Planejamento e Meio Ambiente, e desenvolvidos na Universidade Federal do Tocantins até final de 2006.

Segundo Silveira et al. (2004), a eficiência reprodutiva dos rebanhos é um dos fatores determinantes da eficiência total de produção e deve, portanto, ser considerada como critério de seleção. A medida do perímetro escrotal (PE) é a característica mais utilizada como critério de seleção para melhorar a eficiência reprodutiva em gado de corte (Pereira et al., 2002). Assim, o PE, nos machos, passou a ser uma característica valiosa na seleção dos pais da próxima geração, pois observaram-se correlações positivas com a produção de sêmen, fertilidade e características de produção como o peso, o que, para o criador, é de suma importância, pois quanto mais rápida for a 
taxa de crescimento dos animais, mais curto será o ciclo de seus produtos, podendo reduzir os custos de manutenção na propriedade, principalmente se o sistema de produção for o confinamento. Isso significa que o rendimento deve ser baseado em quantidade de animais vendidos jovens e não em animais vendidos mais velhos e mais pesados (Marcondes, 1999). Pereira et al. (1998) estimaram a herdabilidade da razão perímetro escrotal sobre peso ao sobreano (PE 550/PES 550) como de média a alta magnitude, em animais da raça Nelore pertencentes ao grupo CFM, concluindo que tais estimativas permitiriam boa resposta à seleção. Estes autores ressaltaram ainda que, a utilização desta característica (ou razão de características) permitiria a seleção de animais com maior perímetro escrotal por unidade de peso vivo, evitando-se a escolha de indivíduos com PE elevado apenas porque teriam peso elevado. Ortiz Peña et al. (2001) comentaram a importância de se conhecer as inter-relações entre o perímetro escrotal e a precocidade de crescimento, sugerindo o uso desta característica corrigida para idade e peso ao sobreano.

No melhoramento animal, os parâmetros de dispersão são frequentemente estimados utilizando máxima verossimilhança restrita (REML) (Patterson e Thompson, 1971), sendo introduzidos os métodos Bayesianos por Gianola e Foulley (1983) e Gianola e Fernando (1986) como uma alternativa de estimação. No Brasil, a análise Bayesiana vem sendo recomendada para avaliação genética em melhoramento animal desde 1997 (Magnabosco, 1997; Rosa, 1998).

Blasco (2001) relatou que tanto os métodos frequentistas (REML) quanto os probabilísticos (inferência bayesiana) possuem fundamentações teóricas consistentes e a escolha de um deles depende da capacidade e da facilidade de resolução de cada caso, bem como de preferências pessoais dos pesquisadores interessados.
A REML e a IB podem ser utilizadas para analisar populações submetidas à seleção baseada no valor genético predito por BLUP ou no valor fenotípico (Van Tassell et al., 1995; Duangjinda et al., 2001; Toral et al., 2007).

A inferência bayesiana propicia estimativas mais precisas de componentes de variância, parâmetros genéticos, valores genéticos e ganhos genéticos, especialmente em amostras de tamanho finito (Resende e Rosa-Perez, 1999), tornando mais uma opção para solução dos problemas relacionados à avaliação genética animal.

Segundo Magnabosco et al. (2001), a aplicação de métodos de Markov Chain Monte Carlo, destacando-se a amostragem de Gibbs, pode ser utilizada como ferramenta para estimação de componentes de covariância e parâmetros genéticos utilizando dados de campo.

Todavia, as estimativas de erro podem ser menores para os componentes obtidos por inferência bayesiana (Van Tassell et al., 1995), em virtude da influência da distribuição a priori sobre os componentes de co-variância a posteriori, porém essas diferenças diminuem quando o número de animais envolvidos nas análises aumenta (Toral et al., 2007). Segundo esses autores, a utilização da inferência bayesiana permite obter, além das estimativas, o intervalo de credibilidade para a distribuição a posteriori dos componentes de co-variância e pode ser mais vantajosa que a REML quando o arquivo de dados é muito grande e os modelos são bastante complexos (Van Tassell et al., 1995; Van Tassell e Van Vleck, 1996).

Atualmente a pecuária brasileira passa da fase de aumento do quantitativo de rebanho, através da incorporação de novas tecnologias de produtos (melhoramento genético e técnicas mais avançadas de processos, como manejo rotacionado/ confinamento) para a fase de aumento de produtividade e rentabilidade, por meio de boas práticas de gestão e controle rigoroso 


\section{MARCONDES, ARAÚJO, VOZZI, GUNSKI, GARNERO E LÔBO}

de custos. Assim, muda-se a estrutura de lucratividade, reformulando todo o processo de obtenção do custo de produção da pecuária, levando em consideração não o lucro/unidade, mas sim o lucro/atividade, onde mais $\mathrm{kg} / \mathrm{ano}$, com um lucro unitário menor, é melhor do que a tradicional visão de lucro/kg (Seagro, 2007). Neste contexto, objetivou-se com este trabalho estimar os parâmetros genéticos do índice perímetro escrotal/peso padronizado para 365 dias de idade (IND365), via inferência bayesiana e sob dois modelos de análise distintos, em animais da raça Nelore criados no Estado do Tocantins, Brasil.

\section{MATERIALEMÉTODOS}

O arquivo inicial de dados continha 14455 animais nascidos no período de 1971 a 2005 em cinco fazendas tocantinenses participantes do Programa de Melhoramento Genético da Raça Nelore (PMGRN - Nelore Brasil). Além de serem identificados de forma a garantir as informações de genealogia, os indivíduos foram submetidos a mensurações trimestrais de peso e perímetro escrotal (PE). A seguinte fórmula foi utilizada para a obtenção do peso à idadepadrão (365 dias):

Peso à idade-padrão $=\mathrm{Pa}+(\mathrm{GMD} x \mathrm{da})$

em que:

Pa é peso anterior à idade-padrão;

GMD é o ganho de peso médio diário;

da são os dias compreendidos entre a pesagem anterior e a idade-padrão.

Os produtos foram monitorados do nascimento aos 21 meses de idade e as matrizes também pesadas ao parto, na desmama dos seus produtos, e nos meses de abril e outubro de cada ano. A desmama dos bezerros é feita geralmente na idade de sete a oito meses. Do banco de dados inicial, 3299 animais apresentavam registros de peso padronizado aos 365 dias de idade (P365) e 851 machos possuíam também o registro do
PE aos 365 dias de idade (PE365).

O índice perímetro escrotal/peso (IND365) foi obtido por meio da razão do PE365 e do P365. Na fase inicial, o banco de dados foi manipulado sob o programa computacional SAS (2001) que serviu para a consistência dos dados, para as estatísticas básicas e para a obtenção dos valores iniciais. Na consistência dos dados, foram eliminados registros fora da margem de três vezes o valor do desvio-padrão, que seriam valores superestimados ou subestimados o que comprometeria a confiabilidade da análise de variância. Realizou-se a análise de variância por meio do programa SAS, PROC GLM, considerando-se no modelo como efeito fixo a classe de idade da vaca ao parto (CIVP), com seis níveis assim distribuídos: vacas com menos de 36 meses, classe 1; entre 36 e 47 meses, classe 2 ; entre 48 e 59 meses, classe 3 ; entre 60 e 71 meses, classe 4; entre 72 e 119 meses, classe 5 e acima de 119 meses, classe 6. Outros efeitos fixos presentes no modelo foram o ano de nascimento (com 10 níveis para IND365), a estação de nascimento (1 para animais nascidos entre janeiro e junho e 2 para aqueles nascidos entre julho e dezembro), fazenda e mês de nascimento. Os grupos de contemporâneos (GC) formaram-se a partir

Tabela I. Estatística descritiva da mensuração de perímetro escrotal ao ano de idade (PE365), do peso ao ano de idade (P365) e do índice perímetro escrotal/peso (IND365), no arquivo de dados original. (Descriptive statistics for scrotal circumference at 365 days of age (PE365), weight at 365 days of age (P365) and scrotal circumference/weight index (IND365)).

\begin{tabular}{lccccc}
\hline Característica & $\mathrm{N}$ & Média & DP & Min & Max \\
PE365 $(\mathrm{mm})$ & 851 & 181 & 20,4 & 120 & 296 \\
P365 $(\mathrm{kg})$ & 3299 & 214 & 41,7 & 81 & 464 \\
IND365 $(\mathrm{mm} / \mathrm{kg})$ & 851 & 0,86 & 0,121 & 0,54 & 1,47
\end{tabular}

$\mathrm{N}$ = número de observações; $\mathrm{DP}=$ desvio-padrão; Min = valor mínimo; Max = valor máximo. 
das informações de fazenda, ano de nascimento, mês de nascimento e lote de manejo aos 365 dias de idade (Araújo et al., 2006) para o IND365. Restaram no arquivo final de dados, 693 registros e 16 GCs.

Para a obtenção dos componentes de variância foram utilizados programas de análise em linguagem Fortran(Misztál, 2001), sendo formatados arquivos de parâmetros para os dois modelos estudados: com e sem a inclusão do efeito materno no modelo, sendo a covariância aditiva-materna fixada como zero, seguindo-se o padrão de análises adotado pelo PMGRN-Nelore Brasil e com valores iniciais obtidos anteriormente por meio do programa MTDFREML - multiple trait derivative free restricted maximum likelihood(Boldman et al., 1995). Em seguida, o arquivo de parâmetros foi submetido ao programa GIBBSF90.exe, que usa metodologia bayesiana com amostragem de Gibbs para características contínuas, assumindo-se distribuições iniciais para efeitos genéticos e residuais com distribuições normais multivariadas, como descrito a seguir:

$$
\begin{aligned}
& \mathrm{P}\left(\mathrm{a} \mid \sigma^{2} \mathrm{a}\right) \sim \mathrm{N}\left(0, \mathrm{~A} \sigma^{2} \mathrm{a}\right) \\
& \mathrm{P}\left(\mathrm{e} \mid \sigma^{2} \mathrm{e}\right) \sim \mathrm{N}\left(0, \mathrm{l} \sigma^{2} \mathrm{e}\right)
\end{aligned}
$$

em que:

A é a matriz de parentesco;

$\sigma^{2} a$ é a variância genética aditiva;

$\sigma^{2}$ e é a variância residual;

I é uma matriz identidade de ordem $r$ por $r$.

Uma análise inicial mostrou que o período de queima não precisaria ser conservador, pois a partir da amostra de número 50 , as estimativas tornavam-se mais estáveis. Implementaram-se um milhão de rodadas e 20000 de período de queima, com amostragem a cada 100 rodadas, definida por Marcondes et al. (2008) no programa POSTGIBBSF90. As saídas passaram a ser trabalhadas no SAS, para calcular as estimativas de herdabilidade $\left(h^{2}\right)$, segundo as fórmulas clássicas. Os arquivos gerados
Tabela II. Correlação serial, média, moda, mediana, valor mínimo e valor máximo das variâncias genéticas aditivas $\left(\sigma^{2} a\right)$ e das estimativas de herdabilidade $\left(h^{2}\right)$ obtidas sob dois modelos (com e sem efeito materno) para a característica IND365. (Serial correlation, mean, mode, median, minimum and maximum values to additive genetic variance $\left(\sigma^{2} \mathrm{a}\right)$ and estimates of heritability $\left(\mathrm{h}^{2}\right)$ obtained from two models (with and without maternal effect) for the

\begin{tabular}{|c|c|c|}
\hline & \multicolumn{2}{|c|}{ Modelo } \\
\hline & SEM & CEM \\
\hline \multicolumn{3}{|c|}{ Variância genética aditiva $\left(\sigma^{2} a\right)$} \\
\hline Correlação serial & 0,803 & 0,792 \\
\hline Média & 0,002 & 0,002 \\
\hline Moda & 0,001 & 0,001 \\
\hline Mediana & 0,002 & 0,002 \\
\hline Mínimo & 0,000 & 0,000 \\
\hline Máximo & 0,010 & 0,009 \\
\hline Curtose & 2,735 & 2,792 \\
\hline Assimetria & 1,232 & 1,250 \\
\hline \multicolumn{3}{|c|}{ Herdabilidade direta $\left(h^{2}\right)$} \\
\hline Média & 0,178 & 0,175 \\
\hline Moda & 0,129 & 0,149 \\
\hline Mediana & 0,162 & 0,161 \\
\hline Mínimo & 0,014 & 0,009 \\
\hline Máximo & 0,858 & 0,722 \\
\hline Curtose & 1,580 & 1,545 \\
\hline Assimetria & 1,023 & 1,003 \\
\hline \multicolumn{3}{|c|}{ Variância materna $\left(\sigma^{2} \mathrm{~m}\right)$} \\
\hline Correlação serial & & 0,828 \\
\hline Média & & 0,001 \\
\hline Moda & & 0,001 \\
\hline Mediana & & 0,001 \\
\hline Mínimo & & 0,000 \\
\hline Máximo & & 0,006 \\
\hline Curtose & & 1,125 \\
\hline Assimetria & & 0,991 \\
\hline \multicolumn{3}{|c|}{ Herdabilidade materna $\left(h^{2} m\right)$} \\
\hline Média & & 0,139 \\
\hline Moda & & 0,086 \\
\hline Mediana & & 0,125 \\
\hline Mínimo & & 0,004 \\
\hline Máximo & & 0,574 \\
\hline Curtose & & 0,676 \\
\hline Assimetria & & 0,881 \\
\hline
\end{tabular}
trait IND365).

SEM: Sem efeito materno; CEM: Com efeito materno. 


\section{MARCONDES, ARAÚJO, VOZZI, GUNSKI, GARNERO E LÔBO}

Tabela III. Região de credibilidade (RC) a 95\% para as variâncias genéticas aditivas $\left(\sigma^{2} a\right)$, herdabilidades direta $\left(h^{2} a\right)$ e materna $\left(h^{2} m\right)$ obtidas sob dois modelos (com e sem efeito materno) para a característica IND365. (Credible region $(\mathrm{RC})$ at $95 \%$ to additive genetic variance $\left(\sigma^{2} a\right)$, direct heritability $\left(h^{2} a\right)$ and maternal $\left(h^{2} m\right)$ obtained from two models (with and without maternal effect) for the trait IND365).

\begin{tabular}{llll}
\hline Modelo & RC a $95 \%\left(\sigma^{2} a\right)$ & RC a $95 \%\left(h^{2} a\right)$ & RC a $95 \%\left(h^{2} m\right)$ \\
\hline Sem efeito materno & 0,00 a 0,004 & 0,049 a 0,397 & - \\
Com efeito materno & 0,00 a 0,004 & 0,048 a 0,380 & 0,03 a 0,323 \\
\hline
\end{tabular}

pelo SAS foram submetidos às análises $a$ posteriori do programa GIBANAL.exe(Van Kaam, 1997), incluindo-se a correlação serial entre as amostras e a obtenção das densidades marginais posteriores para confecção dos gráficos de distribuição posterior das herdabilidades, em Excel for Windows.

\section{RESULTADOSEDISCUSSÃO}

A tabela I apresenta a descrição dos registros referentes à padronização para 365 dias de idade, antes da consistência dos dados. Observa-se que somente $26 \%$ dos machos tiveram, pelo menos, duas mensurações de PE para que fosse possível a padronização do PE365. Com isto, ações de esclarecimento sobre a importância da medida, sua obtenção e controle da qualidade das mensurações a campo poderiam ser direcionadas especificamente aos criadores do Estado do Tocantins. O valor médio do IND365 foi superior àquele encontrado por Pereira et al. (1998), de 0,02 $\mathrm{cm} / \mathrm{kg}$ para animais pertencentes a outra região brasileira, ou seja, para os animais do Tocantins a cada $1 \mathrm{~kg}$ de peso observou-se $0,86 \mathrm{~mm}$ de PE. O uso de poucos reprodutores, a entrada recente no programa de melhoramento e a baixa qualidade do controle zootécnico, sendo poucos animais pesados e medidos (683 animais), em relação aos 14455 nascidos, são fatores que afetam diretamente a qualidade dos arquivos de desempenho e de genealogia e, consequentemente, a detecção de variabilidade nos rebanhos estudados.

Os resultados obtidos para os dois modelos implementados estão sumarizados nas tabelas II e III. Observa-se alta correlação serial entre as amostras, valores médios e medianas semelhantes entre os dois modelos, com pequena variação nos valores mínimos e máximos, as quais também podem ser evidenciadas graficamente. As altas correlações seriais observadas na análise indicaram o baixo valor informativo das amostras, necessitando da análise pelo Gibanal para obtenção de período adicional de queima e/ou skip time, os quais reduzem o número de amostras a serem trabalhadas, porém as amostras restantes são mais informativas.

As regiões de credibilidade a $95 \%$ foram semelhantes entre os modelos estudados (tabela III), destacando-se, no entanto, valores expressivos para a estimativa de herdabilidade materna (entre 0,03 e 0,32).

Após o período adicional de queima e o skip time sugerido pelo programa Gibanal para o modelo sem efeito materno (respectivamente, 16 e 19), as correlações seriais para $\sigma^{2} \mathrm{a}$ e $\mathrm{h}^{2}$ ficaram em 0,07 restando 526 amostras para serem trabalhadas. Para o modelo com efeito materno, o programa sugeriu uma queima adicional de 10 amostras e skip time de 19, restando igualmente 526 amostras com correlação serial de $-0,01$. Considerando que a correlação serial indica o grau de informação fornecido por cada amostra para a média a posteriori, ou seja, refere-se à parte da medição das amostras 
Tabela IV. Média, moda, mediana, valor mínimo e valor máximo das variâncias genéticas aditivas $\left(\sigma^{2}\right.$ a) e das estimativas de herdabilidade $\left(h^{2}\right)$ obtidas sob dois modelos (com e sem efeito materno) para a característica IND365, após análise do programa Gibanal. (Mean, mode, median, minimum and maximum values to additive genetic variance $\left(\sigma^{2} a\right)$ and estimates of heritability $\left(\mathrm{h}^{2}\right)$ obtained from two models - with and without maternal effect - for the trait IND365 after analysis in Gibanal program).

\begin{tabular}{|c|c|c|}
\hline Modelo & Sem efeito materno & Com efeito materno \\
\hline \multicolumn{3}{|c|}{ Variância genética aditiva $\left(\sigma^{2} a\right)$} \\
\hline Correlação serial & 0,069 & 0,067 \\
\hline Média $\pm \mathrm{MCE}^{\star}$ & $0,002 \pm 0,00003$ & $0,002 \pm 0,00003$ \\
\hline Moda & 0,001 & 0,001 \\
\hline Mediana & 0,002 & 0,002 \\
\hline Mínimo & 0,000 & 0,000 \\
\hline Máximo & 0,005 & 0,007 \\
\hline Curtose & 1,159 & 3,057 \\
\hline Assimetria & 1,009 & 1,324 \\
\hline \multicolumn{3}{|c|}{ Herdabilidade direta $\left(h^{2}\right)$} \\
\hline Média $\pm S D^{\star \star}$ & $0,175 \pm 0,087$ & $0,180 \pm 0,089$ \\
\hline Moda & 0,132 & 0,145 \\
\hline Mediana & 0,163 & 0,166 \\
\hline Mínimo & 0,022 & 0,011 \\
\hline Máximo & 0,515 & 0,642 \\
\hline Curtose & 0,756 & 1,741 \\
\hline Assimetria & 0,890 & 1,030 \\
\hline \multicolumn{3}{|c|}{ Variância materna $\left(\sigma^{2} \mathrm{~m}\right)$} \\
\hline Correlação serial & & $-0,015$ \\
\hline Média \pm MCE & & $0,001 \pm 0,00003$ \\
\hline Moda & & 0,001 \\
\hline Mediana & & 0,001 \\
\hline Mínimo & & 0,000 \\
\hline Máximo & & 0,004 \\
\hline Curtose & & 0,600 \\
\hline Assimetria & & 0,948 \\
\hline \multicolumn{3}{|c|}{ Herdabilidade materna $\left(\mathrm{h}^{2} \mathrm{~m}\right)$} \\
\hline Média $\pm S D$ & & $0,143 \pm 0,082$ \\
\hline Moda & & 0,097 \\
\hline Mediana & & 0,123 \\
\hline Mínimo & & 0,007 \\
\hline Máximo & & 0,399 \\
\hline Curtose & & 0,245 \\
\hline Assimetria & & 0,836 \\
\hline
\end{tabular}

${ }^{\star}$ Erro de Monte Carlo; ** Desvio-padrão.

relacionadas ao tempo de variação do efeito estocástico causador da correlação entre as observações dentro de um curto intervalo de tempo, tal efeito e menos correlacionado quando as medições forem mais espaçadas, assim deseja-se que as amostragens forneçam a menor correlação possível (Yamaki, 2006). Neste sentido, a análise 


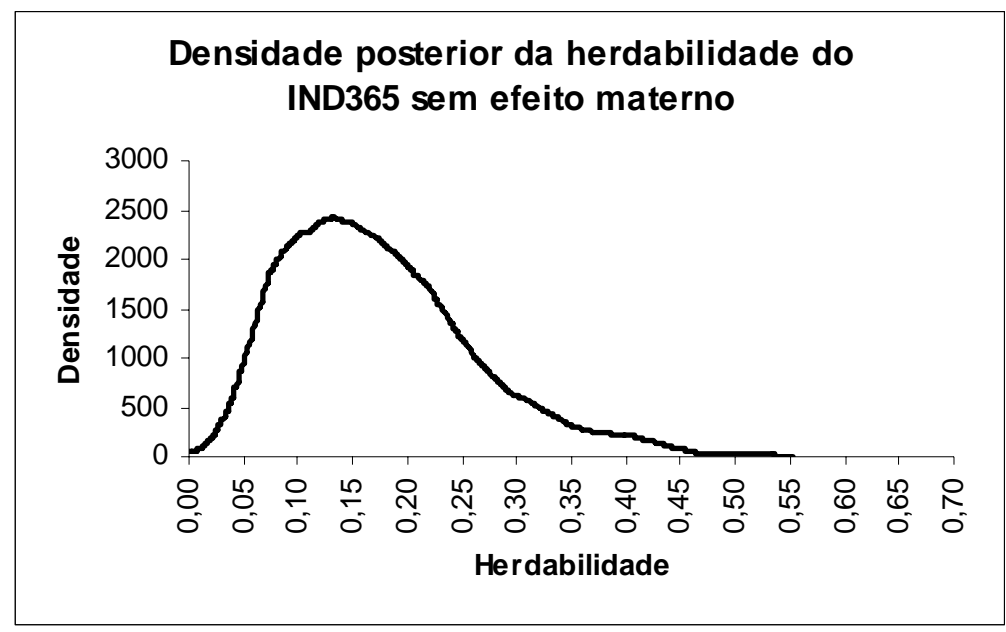

Figura 1. Distribuição amostral das estimativas de herdabilidade direta obtidas sob modelo sem efeito materno para a característica IND365. (Distribution sample of heritabilities obtained under direct model without maternal effect for trait IND365).

adotada foi suficiente para atingir os critérios de convergência e para minimizar os efeitos causados por esta variação. Os valores recalculados no programa encontram-se na tabela IV.
Houve alteração nos valores mínimos e máximos da variância aditiva e das estimativas de herdabilidade, nos dois modelos estudados, quando são observadas as tabelas II e IV. A tabela IV indica, por meio

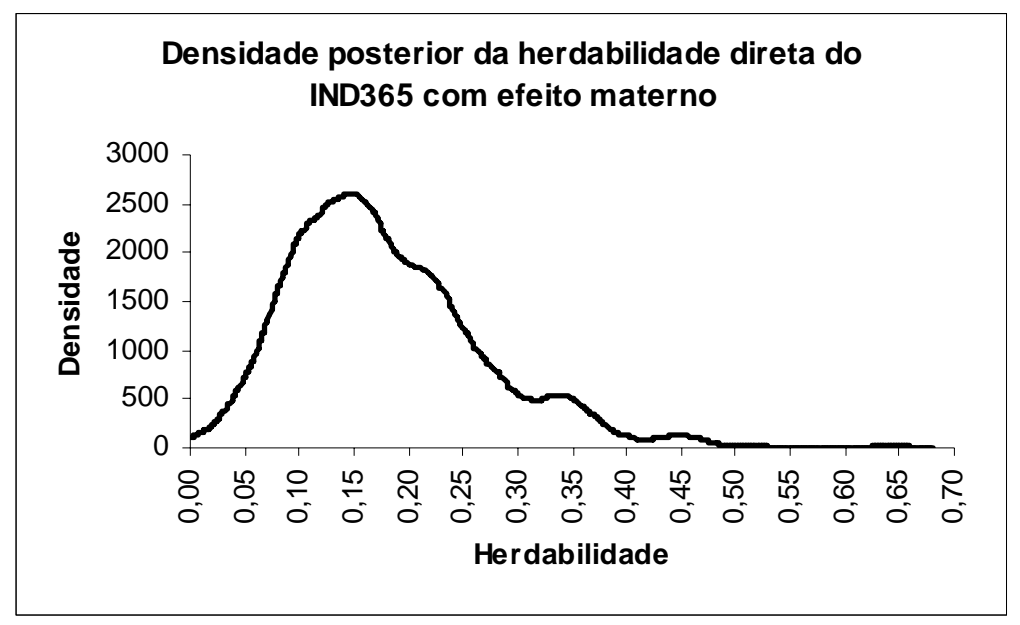

Figura 2. Distribuição amostral das estimativas de herdabilidade direta obtidas sob modelo com efeito materno para a característica IND365. (Distribution sample of heritabilities obtained under direct model with maternal effect for trait IND365). 


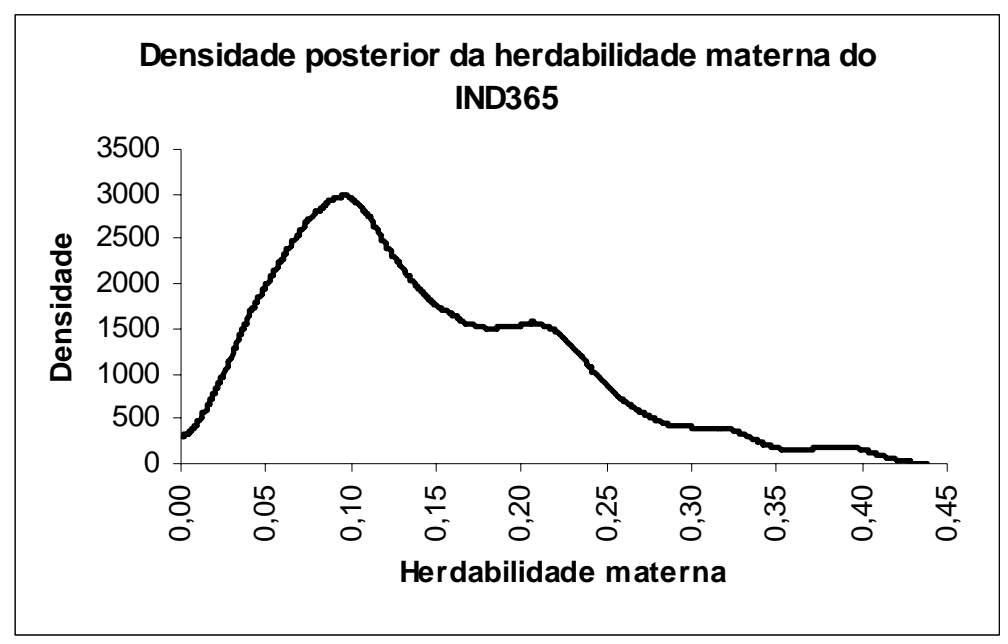

Figura 3. Distribuição amostral das estimativas de herdabilidade materna obtidas sob modelo com efeito materno para a característica IND365. (Distribution sample of maternal heritabilities obtained under direct model with maternal effect for trait IND365).

dos valores de assimetria e curtose que houve alteração no grau de achatamento da distribuição da $\sigma^{2}$ a, quando foi considerado no modelo o efeito materno, passando de platicúrtica a leptocúrtica. Todas as demais estimativas, no entanto, tiveram distribuições assimétricas positivas e platicúrticas. As figuras 1 a 3 ilustram graficamente a distribuição das estimativas de herdabilidade direta e materna obtidas para a característica IND365, neste estudo.

Comparando-se os dois gráficos de distribuição das estimativas de herdabilidade direta, observa-se que aquela obtida por meio do modelo que considerava o efeito materno apresentou uma distribuição trimodal mais evidente, com uma segunda moda entre 0,20 e 0,25 e uma terceira moda próxima ao valor de 0,35 . Tendência semelhante ocorreu na distribuição das estimativas de herdabilidade materna, apresentando-se bimodal, com picos próximos aos valores de 0,10 e 0,20. Foi interessante a obtenção de estimativas de herdabilidade materna tão expressivas para uma característica mensurada entre quatro a seis meses após a desmama, evidenciando-se a influência da habilidade maternal ainda no desenvolvimento pós-desmama de suas crias.

Os valores encontrados via inferência bayesiana estão próximos àqueles obtidos por Araújo et al. (2010), porém observa-se que a variabilidade foi melhor detectada por esta metodologia e, se o valor da moda for considerado $(0,13$ e 0,15$)$, este apresentouse superior àqueles obtidos pelos referidos autores. Glória et al. (2006), estimaram as herdabilidades das características P365 e PE365 na mesma base de dados, obtendo os valores de 0,19 e 0,17 , em análise bi-característica. No entanto, comparando-se com a estimativa de Pereira et al. (1998) obtida pelo programa MTDFREML (Boldman et al., 1995), de 0,34, apresentaram-se inferiores. Esta diferença pode ser atribuída ao fato dos criadores tocantinenses utilizarem, basicamente, os mesmos reprodutores e, ainda, ao tamanho da população estudada (com reduzido número de machos mensurados quanto ao perímetro escrotal). A comparação com outros estudos não é 


\section{MARCONDES, ARAÚJO, VOZZI, GUNSKI, GARNERO E LÔBO}

possível, pois não são encontrados outros relatos para esta característica.

\section{CONCLUSÕES}

As estimativas de herdabilidade obtidas para o IND365, semelhantes àquelas obtidas para as características peso e perímetro escrotal, em separado, para a mesma população estudada de animais Nelore da região Norte brasileira, sugerem variabilidade genética para responder de forma satisfatória ao processo seleção, tornando um critério alternativo viável para os programas de melhoramento genético. Pode-se proceder a uma implementação com cadeia longa, tomada de amostra a cada 100 ou 500

\section{BIBLIOGRAFIA}

Araújo, R.O., Marcondes, C.R., Gunski, R.J. e Lôbo, R.B. 2006. Análises preliminares do índice perímetro escrotal/peso padronizado como critério de seleção para a raça Nelore. In: Seminário de Iniciação Científica da UFT, 2. Anais... Palmas. Brasil.

Araújo, R.O., Glória, W.P., Marcondes, C.R., Garnero, A. del V., Gunski, R.J., e Lôbo, R.B. 2010. Índice perímetro escrotal-peso ajustado aos 365 e 450 dias de idade para bovinos Nelore. Rev. Ciênc. Agr., 53: 171-176.

Blasco, A. 2001. The bayesian controversy in animal breeding. J. Anim. Sci., 79: 20232046.

Boldman, K.G., Kriese, L.A., Van Vleck, L.D., Van Tassell, C. and Kachman, S.D. 1995. A manual for use of MTDFREML. A set of programs to obtains estimates of variances and covariances [DRAFT]. U.S. Departament of Agriculture. Agricultural Research Service.

Boligon, A.A., Albuquerque, L.G., Rorato, P.R.N. 2008. Associações genéticas entre pesos e características reprodutivas em rebanhos da raça Nelore. Rev. Bras. Zootecn., 37: 596-601.

Cyrillo, J.N.S.G., Alencar, M.M., Razook, A.G., Mercadante, M.E.Z. e Figueiredo, L.A. 2004. Modelagem e estimação de parâmetros genéticos e fenotípicos para pesos do nascimento à seleção (378 dias) de machos Nelore. Rev. rodadas e um burn-in pouco conservador, para uma análise sob inferência Bayesiana e modelo animal da característica IND365 e com limitada estrutura de dados

\section{AGRADECIMENTOS}

O presente trabalho foi realizado com o apoio do Conselho Nacional de Desenvolvimento Científico e Tecnológico CNPq - Brasil, em parceria com a Secretaria de Ciência e Tecnologia do Estado do Tocantins - SECT, do Programa de Melhoramento Genético da Raça Nelore (PMGRN- Nelore Brasil) e da Associação Nacional de Criadores e Pesquisadores (ANCP).

Bras. Zootecn., 33: 1405-1415.

Duangjinda, M., Misztal, I., Bertrand, J.K. and Tsuruta, S. 2001. The empirical bias of estimates by restricted maximum likelihood, bayesian method, and method $\mathrm{R}$ under selection for additive, maternal, and dominance models. $J$. Anim Sci., 79: 2991-2996.

Gianola, D. and Foulley, J.L. 1983. Sire evaluation for ordered categorical data with a threshold model. Genet Sel. Evol., 15: 201-224.

Gianola, D. and Fernando, R.L. 1986. Bayesian methods in animal breeding theory. J. Anim. Sci., 63: 217-244.

Glória, W.P., Araújo, R.O., Marcondes, C.R., Garnero, A.V. e Lôbo, R.B. 2006. Características correlacionadas de peso e perímetro escrotal pós-desmama de animais tocantinenses da raça Nelore: análises iniciais. In: Seminário de Iniciação Científica da UFT, 2. Anais... Palmas-TO.

Gunski, R.J., Garnero, A. del V., Reyes, A. de los, Bezerra, L.A.F. e Lôbo, R.B. 2001. Estimativas de parâmetros genéticos para características incluídas em critérios de seleção em gado Nelore. Ciên. Rural, 31: 603-607.

Magnabosco, C. de U. 1997. Estimativa de parâmetros genéticos em características de crescimento de animais da raça Nelore usando os métodos de máxima verossimilhança restrita 


\section{ÍNDICE PERÍMETRO ESCROTAL/PESO EM REBANHOS NELORE}

e amostragem de Gibbs. Tese (Doutorado em Ciências Biológicas). Faculdade de Medicina de Ribeirão Preto. Universidade de São Paulo. Ribeirão Preto. 56 pp.

Magnabosco, C. de U., Faria, C. de U., Reyes, A. de los, Lôbo, R.B. e Sainz, R.D. 2001. Implementação da amostragem de Gibbs para a estimação de componentes de co-variância e parâmetros genéticos em dados de campo de bovinos Nelore. Embrapa Cerrados. Planaltina. 71: 1-50.

Marcondes, C.R. 1999. Análises de alguns critérios de seleção em gado de corte visando precocidade de crescimento na raça Nelore. Dissertação (Mestrado em Zootecnia). Escola de Veterinária da Universidade Federal de Minas Gerais. Belo Horizonte. 71 pp.

Marcondes, C.R., Araújo, R.O., Vozzi, P.A., Gunski, R.J., Garnero, A. del V., Bezerra, L.A.F. e Lôbo, R.B. 2008. Estudo do período de aquecimento e tomada de amostra para característica que associa peso e perímetro escrotal na raça Nelore. Simpósio Nacional da Sociedade Brasileira de Melhoramento Animal, 7. Anais... São Carlos.

Martins Filho, R., Santos, P.F., Malhado, C.H.M., Carneiro, P.L.S. e Santos, L.H. 2005. Correlação genética, fenotípica e ambiental do desenvolvimento ponderal em bovinos da raça Nelore mocha. In: Reunião Anual da Sociedade Brasileira de Zootecnia, 42. Anais... UFG. Goiânia.

Mercadante, M. E. Z., Lôbo, R.B., Reyes, A. de los, e Duarte, A.M.D. 1997. Estimativas de covariância para crescimento e eficiência reprodutiva em fêmeas de um rebanho Nelore. In: Reunião Anual da Sociedade Brasileira de Zootecnia, 34. Anais... Juiz de Fora. pp. 180182.

Misztál, I. Computer programs. 2001. Disponível em: http://nce.ads.uga.edu/ ignacy/programs. html (20/04/10).

Ortiz Peña, C. D., Queiroz, S.A. e Fries, L.A. 2001. Comparação entre critérios de seleção de precocidade sexual e a associação destes com características de crescimento em bovinos Nelore. Rev. Bras. Zootecn., 30: 93-100.

Patterson, H.D. and Thompson, R. 1971. Recovery of inter-block information when block sizes are unequal. Biometrics, 58: 545.

Pereira, E., Eler, J.P. e Ferraz, J.B.S. 1998. Análise genética da relação perímetro escrotal (PE 550)/ peso aos 550 dias (PES 550) na raça Nelore. Simpósio da Sociedade Brasileira de Melhoramento Animal, 2. Anais... Uberaba. pp. 325-326.

Pereira, E., Eler, J.P. e Ferraz, J.B.S. 2000. Correlação genética entre perímetro escrotal e algumas características reprodutivas da raça Nelore. Rev. Bras. Zootecn., 29: 1678-1683.

Pereira, E., Eler, J.P. e Ferraz, J.B.S. 2002. Análise genética de características reprodutivas na raça Nelore. Pesq. Agropec. Bras., 37: 703-708.

Resende, M.D.V. e Rosa-Perez, J.R.H. 1999. Genética quantitativa e estatística no melhoramento animal. UFPR. Curitiba. $494 \mathrm{pp}$.

Rosa, G.J.M. 1998. Análise bayesiana de modelos lineares mistos robustos via amostrador de Gibbs. Piracicaba. Tese (Doutorado em Zootecnia). Escola Superior de Agricultura Luiz de Queiroz. Universidade Estadual de São Paulo. Piracicaba. 45 pp.

SAS. 2001. Statistical analysis system. User guide: Stat. V. 8.2. SAS Institute Inc. Cary. NC.

Seagro. 2007. Disponível em: http://www.seagro. to.gov.br/noticia.php?id=29 (06/10/08).

Seagro. 2010. Disponível em: http://www.seagro. to.gov.br/noticia.php?id=1462 (20/04/10).

Silveira, J. C., Mcmanus, C., Mascioli, A.S., Silva, L.O.C., Silveira, A.C., Garcia, J.A.S. e Louvandini, H. 2004. Fatores ambientais e parâmetros genéticos para características produtivas e reprodutivas em um rebanho Nelore no Estado do Mato Grosso do Sul. Rev. Bras. Zootecn., 33: 1432-1444.

Toral, F.L.B., Alencar, M.M. e Freitas, A.R. 2007. Abordagens frequentista e bayesiana para avaliação genética de bovinos da raça Canchim para características de crescimento. Rev. Bras. Zootecn., 36: 43-53.

Van Kaam, J.B.C.H.M. 1997. Gibanal. Analyzing program for Markov Chain Monte Carlo sequences, version 2.3. Department of Animal Breeding. Wageningen. The Netherlands.

Van Tassel, C.P., Casella, G. and Pollak, E.J. 1995. Effects of selection on estimates of variance components using Gibbs sampling and restricted maximum likelihood. J. Dairy Sci., 78: 678.

Van Tassel, C.P. and Van Vleck, L.D. 1996. Multipletrait Gibbs sampler for animal models: flexible programs for Bayesian and likelihood-based 


\section{MARCONDES, ARAÚJO, VOZZI, GUNSKI, GARNERO E LÔBO}

(co)variance component inference. J. Anim. Sci., 74: 2586-2597.

Yamaki, M. 2006. Estimação de parâmetros genéticos de produção de leite e de gordura da raça Pardo-suíça utilizando metodologias frequentista e bayesiana. Dissertação (Mestrado em Zootecnia). Universidade Federal de Viçosa.
Viçosa. $64 \mathrm{pp}$.

Yokoo, M.J. I., Albuquerque, L.G., Lôbo, R.B., Sainz, R.D., Júnior, J.M.C., Bezerra, L.A.F. e Araujo, F.R.C. 2007. Estimativas de parâmetros genéticos para altura do posterior, peso e circunferência escrotal em bovinos da raça Nelore. Rev. Bras. Zootecn., 36: 1761-1768. 